\title{
Fibulin-5, an integrin-binding matricellular protein: its function in development and disease
}

\author{
Hiromi Yanagisawa • Marie K. Schluterman • \\ Rolf A. Brekken
}

Received: 24 June 2009 /Accepted: 31 August 2009 /Published online: 2 October 2009

(C) The Author(s) 2009. This article is published with open access at Springerlink.com

\begin{abstract}
Interactions between the extracellular matrix (ECM) and cells are critical in embryonic development, tissue homeostasis, physiological remodeling, and tumorigenesis. Matricellular proteins, a group of ECM components, mediate cell-ECM interactions. One such molecule, Fibulin-5 is a $66-\mathrm{kDa}$ glycoprotein secreted by various cell types, including vascular smooth muscle cells (SMCs), fibroblasts, and endothelial cells. Fibulin-5 contributes to the formation of elastic fibers by binding to structural components including tropoelastin and fibrillin-1, and to cross-linking enzymes, aiding elastic fiber assembly. Mice deficient in the fibulin-5 gene $(F b \ln 5)$ exhibit systemic elastic fiber defects with manifestations of loose skin, tortuous aorta, emphysematous lung and genital prolapse. Although Fbln5 expression is down-regulated after birth, following the completion of elastic fiber formation, expression is reactivated upon tissue injury, affecting diverse cellular functions independent of its elastogenic function. Fibulin-5 contains an evolutionally conserved arginineglycine-aspartic acid (RGD) motif in the N-terminal region,
\end{abstract}

H. Yanagisawa $(\bowtie)$

Department of Molecular Biology,

University of Texas Southwestern Medical Center,

5323 Harry Hines Blvd.,

Dallas, TX 75390-9148, USA

e-mail: hiromi.yanagisawa@utsouthwestern.edu

M. K. Schluterman • R. A. Brekken

Department of Surgery,

Hamon Center for Therapeutic Oncology Research,

University of Texas Southwestern Medical Center,

5323 Harry Hines Blvd.,

Dallas, TX 75390-8593, USA which mediates binding to a subset of integrins, including $\alpha 5 \beta 1, \alpha v \beta 3$, and $\alpha v \beta 5$. Fibulin- 5 enhances substrate attachment of endothelial cells, while inhibiting migration and proliferation in a cell type- and context-dependent manner. The antagonistic function of fibulin- 5 in angiogenesis has been demonstrated in vitro and in vivo; fibulin-5 may block angiogenesis by inducing the anti-angiogenic molecule thrompospondin-1, by antagonizing $\mathrm{VEGF}_{165^{-}}$ mediated signaling, and/or by antagonizing fibronectinmediated signaling through directly binding and blocking the $\alpha 5 \beta 1$ fibronectin receptor. The overall effect of fibulin5 on tumor growth depends on the balance between the inhibitory property of fibulin-5 on angiogenesis and the direct effect of fibulin-5 on proliferation and migration of tumor cells. However, the effect of tumor-derived versus host microenvironment-derived fibulin-5 remains to be evaluated.

Keywords Angiogenesis - Cutis laxa - Elastic fibers ·

Fibulin · Fibronectin · Integrin · ROS · Thrombospondin .

Tumor

$\begin{array}{ll}\text { Abbreviations } \\ \text { ECM } & \text { extracellular matrix } \\ \text { MMP } & \text { matrix metalloproteinase } \\ \text { LTBP } & \text { latent TGF } \beta \text { binding protein } \\ \text { LOXL1 } & \text { lysyl oxidase-like 1 } \\ \text { POP } & \text { pelvic organ prolapse } \\ \text { RGD } & \text { arginine-glycine-aspartic acid } \\ \text { ROS } & \text { reactive oxygen species } \\ \text { TGF- } \beta & \text { transforming growth factor-beta } \\ \text { SMC } & \text { smooth muscle cell } \\ \text { VEGF } & \text { vascular endothelial growth factor }\end{array}$




\section{Introduction}

The extracellular matrix (ECM) is essential for normal development and maintenance of the microenvironment of embryonic and adult tissues. The ECM is composed of structural proteins, glycosaminoglycans, growth factors and cytokines, secreted (pro)enzymes, and glycoproteins called matricellular proteins. The ECM not only provides structural stability to tissues and organs, but also regulates activities of growth factors and cytokines by directly tethering inactive pro-forms to structural components of the ECM (ten Dijke and Arthur 2007). The composition of the ECM has profound effects on cell behavior, including adhesion, proliferation, and migration, as well as on the maintenance of the stem cell niche (Raines 2000; Shen et al. 2008). In addition, matricellular proteins participate in dynamic matrix-cell interactions and fine tune cellular functions (Bornstein 1995).

Over the last two decades, an increasing number of proteins have emerged as matricellular proteins, including thrombospondins and SPARC family members (Bornstein 2001; Brekken and Sage 2001). The fibulin family of ECM proteins has gained attention because of a wide spectrum of cellular functions, as well as essential contributions to the formation of elastic fibers and basement membranes by aiding in assembly and stabilization of macromolecular ECM complexes (reviewed in Argraves et al. 2003; Chu and Tsuda 2004; de Vega et al. 2009). While it is difficult to draw a line precisely between structural proteins and matricellular proteins, we include fibulins as part of the matricellular family because of their diverse cellular and biological functions that involve both matrix and neighboring cells. We will focus on Class II short fibulins, specifically fibulin-5 (also known as EVEC, DANCE) in this review, and discuss domain structure, gene expression, cellular functions, and involvement in various biological processes during development and in pathological conditions.

\section{Fibulin-5: overview}

Fibulin-5 was first identified in 1999 by two groups in search of genes involved in phenotypic modulation of vascular smooth muscle cells (SMCs) and in cardiovascular development through subtractive hybridization and signal sequence trap cloning, respectively (Kowal et al. 1999; Nakamura et al. 1999). Fibulin-5 was shown to be a $66-\mathrm{kDa}$ glycoprotein containing six calcium-binding EGF-like (cbEGF) motifs, which are believed to provide stability and facilitate protein interaction. Fibulin-5 also contains a RGD motif involved in the binding to a subset of cellsurface integrins. In the initial reports, the fibulin-5 gene $(F b \ln 5)$ was shown to be strongly expressed in the embryonic vasculature and neural crest, but was downregulated in all adult tissues, except in the uterus where active remodeling and angiogenesis takes place. Fbln 5 expression, however, was reactivated in injured vessels, including the neointima induced by balloon withdrawal injury, and in atherosclerotic plaques in the mouse model of hypercholesterolemia (Kowal et al. 1999; Nakamura et al. 1999), suggesting a regulatory role in vascular cell function. Fibulin-5 was also identified as a TGF- $\beta$ inducible gene in 3T3-L1 fibroblasts and was shown to induce DNA synthesis in a Smad3-dependent manner. In contrast, fibulin-5 inhibited cell proliferation and cyclin A expression in mink lung epithelial cells, suggesting a potential involvement in the control of cell proliferation in a context-dependent manner (Schiemann et al. 2002).

The biological function of fibulin-5 in vivo, however, was unknown until the generation of Fbln 5 knockout mice, in which systemic elastic fiber defects were revealed (Nakamura et al. 2002; Yanagisawa et al. 2002). Fbln5null mice survive to adulthood but progressively develop severe elastinopathy, including loose skin, tortuous aorta, emphysematous lung, and genital prolapse. These observations establish the first animal model for congenital elastic fiber defects. Fbln5-null mice exhibit an elevated pulse pressure, and $F b \ln 5$-null aortic explants show a significant decrease in extensibility compared to the wild-type vessels, demonstrating that compromised elastic fibers lead to stiff vessels with decreased elasticity. Fbln 5 -null skin and lungs contain only short, disrupted elastic fibers, and no signs of inflammatory infiltrates were observed. In addition, the aorta, lungs, and skin did not show a disruption in collagen fiber formation, confirming that the defects in Fbln5-null mice were confined to the elastic fiber system.

The biological functions of fibulin-5 have been investigated in in vitro studies, and can be segregated into elastogenic and extra-elastogenic functions. However, distinguishing these functions in vivo is challenging due to the fact that insoluble elastin is known to influence cellular behavior (Karnik et al. 2003).

\section{Fibulin-5: an integrin-binding member of the Class II fibulin subfamily}

Fibulins are characterized by tandem repeats of calciumbinding EGF (cbEGF)-like motifs and a globular C-terminal fibulin module. There are seven known fibulins (Fig. 1), which can be subdivided into two subfamilies based on their size and domain structure. The Class I fibulin subfamily includes the prototype fibulin-1, fibulin-2, and fibulin-6 (reviewed in Argraves et al. 2003; Timpl et al. 2003). In this subfamily, cbEGF repeats are longer than in Class II fibulins, and there are additional $\mathrm{N}$-terminal domains that are not 
Fig. 1 Schematic presentation of fibulin family proteins. Class I fibulins include long fibulins (fibulin-1, -2, and -6) and Class II fibulins include short fibulins (fibulin-3, -4, -5, and -7).

Fibulin-5 contains an evolutionally conserved RGD (arginineglycine-asparatic acid) sequence in the first cbEGF motif, whereas the RGD sequence in fibulin-2 is not conserved among the species. Human fibulin-6 with 44 repeats of immunoglobulin domain is shown. Parentheses indicate synonym(s) of the corresponding fibulin shown in the figure

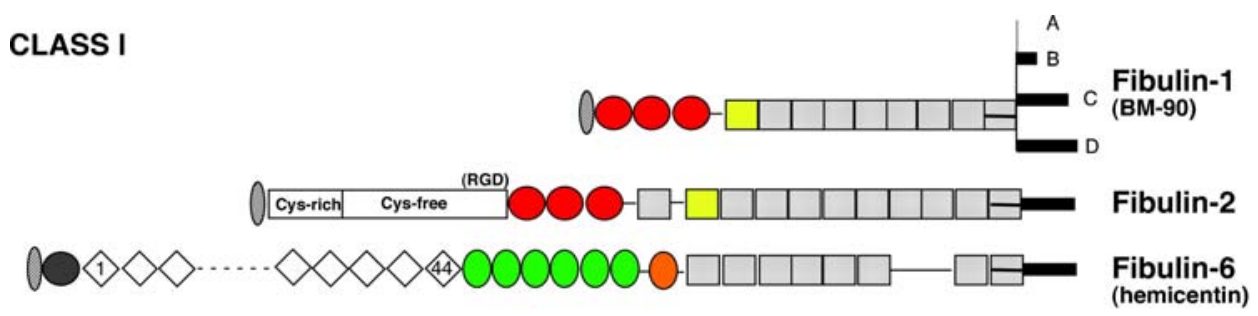

CLASS II

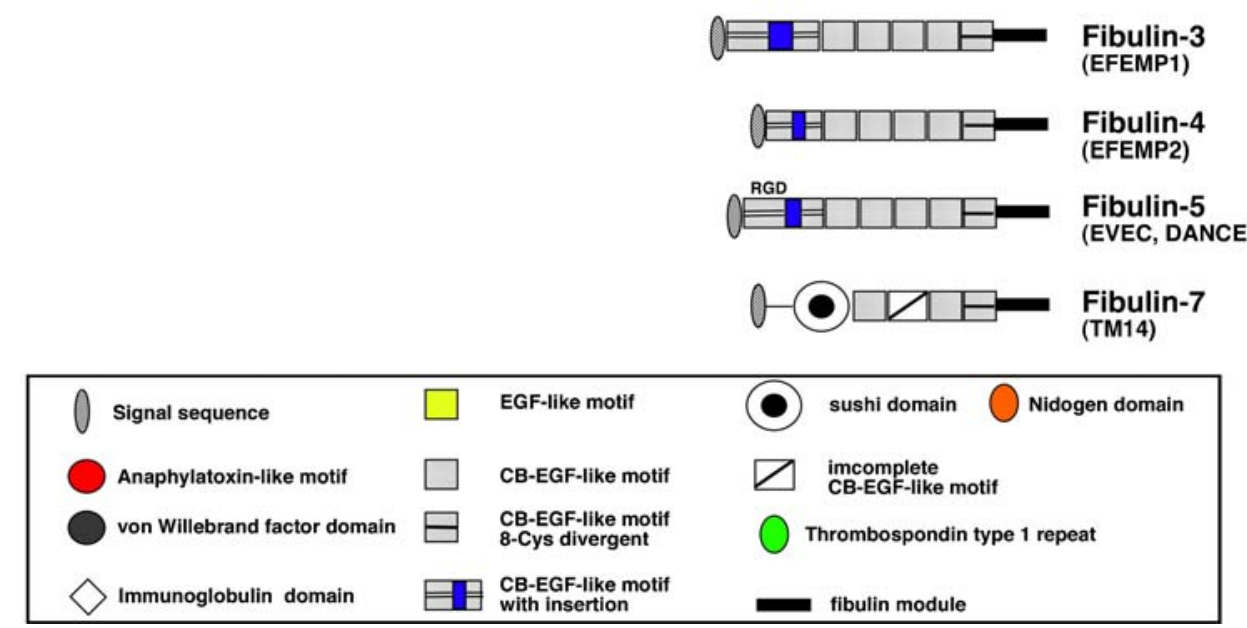

shared by Class II fibulins. Fibulin- 6 contains the largest Nterminal domain, consisting of a von Willebrand factor domain, more than forty immunoglobulin domains depending on the species, and six thrombospondin type I repeats (Vogel and Hedgecock 2001). Class II fibulins include fibulin-3, fibulin-4, fibulin-5 and fibulin-7. Fibulins-3, -4 and -5 contain six cbEGF domains, the first of which contains a proline-rich insertion sequence, and the sixth domain, which is a divergent type with 8 cysteines. The identity score of the primary amino acid sequence between fibulin-5 and fibulins-3, -4 and -7 in humans is $41 \%, 49 \%$, and $24 \%$, respectively. Fibulin- 7 is the newest member and is rather atypical because of the shorter cbEGF domains and the presence of a sushi domain, which is frequently found in complement proteins, but is absent in fibulins-3, -4 , and -5 (de Vega et al. 2007).

Fibulin-5 contains an evolutionally conserved RGD sequence in the first cbEGF domain (Fig. 2). The RGD sequence is present in various matricellular and ECM proteins, including fibronectin, vitronectin, osteopontin and thrombospondins, and is recognized by heteromeric integrin receptors to participate in cellular functions (Davis et al. 2000; Ruoslahti and Pierschbacher 1987). For example, ECM-cell binding is essential for the assembly of fibrous ECM proteins such as fibronectin (Wu et al. 1995), to trigger cellular effects via the cytoplasmic tail of $\beta$ integrin (Legate et al. 2006), and to form cell- surface protease complexes, involving urokinase type plasminogen activator (uPA)/uPA receptor, vitronectin, and activated integrins (Madsen and Sidenius 2008).

Fibulin-5 was shown to mediate binding to human umbilical vein endothelial cells (HUVECs) in a RGDdependent manner (Nakamura et al. 1999). Further, it was shown that the N-terminal half of fibulin-5 mediates cell attachment via $\alpha \mathrm{v} \beta 3, \alpha \mathrm{v} \beta 5$ and $\alpha 9 \beta 1$ integrins (Nakamura et al. 2002). On the other hand, Lomas et al. found that fibulin-5 mediates attachment and spreading of primary aortic SMCs through binding to the fibronectin receptor $\alpha 5 \beta 1$ and $\alpha 4 \beta 1$, but not to $\alpha \mathrm{v} \beta 3$ (Lomas et al. 2007). Although the RGD motif and insertion sequence of fibulin5 was suggested to be exposed to the cell surface (Albig and Schiemann 2005), direct protein interaction assays revealed that fibulin- 5 was only able to bind to $\alpha \mathrm{v} \beta 3$ after reduction and alkylation, which unmasks the RGD sequence (Kobayashi et al. 2007). Furthermore, truncated fibulin-5, containing the first cbEGF domain alone, did not support binding and spreading of SMCs. Taken together, these results indicate that fibulin-5-integrin interactions may require efficient exposure of the RGD motif and presence of the flanking domains of fibulin-5 (Lomas et al. 2007). Interestingly, fibulin-5 failed to activate downstream signaling after binding to $\alpha 5 \beta 1$ and $\alpha 4 \beta 1$ integrins. Fibulin- 5 antagonized fibronectin-induced stress fiber formation and focal adhesions in SMCs in a dose-dependent manner, suggesting that fibulin-5 acts in a dominant-negative fashion to inhibit fibronectin receptor-mediated signaling. 

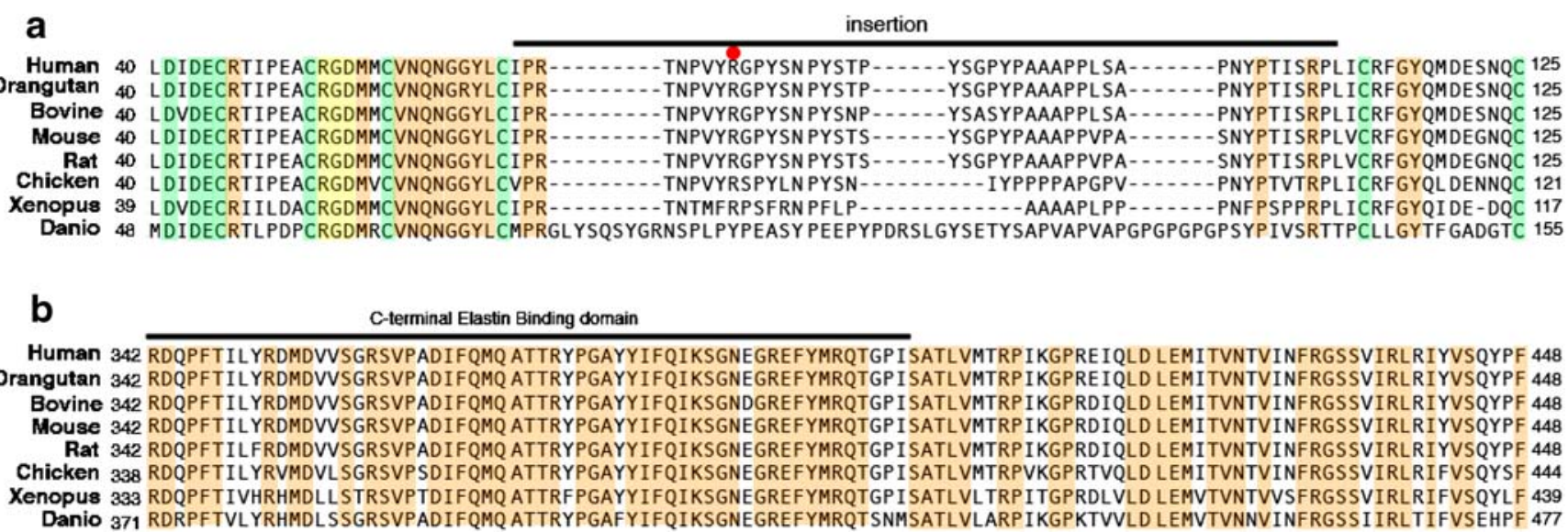

Fig. 2 Alignment of fibulin-5 from Human, Orangutan, Bovine, Mouse, Rat, Chicken, Xenopus, and Danio in the first calcium binding EGF-like domain with insertion a and a C-terminal fibulin module b. Green shade represents a consensus of calcium binding motifs, yellow shade represents the RGD sequence, and orange shade represents a conserved sequence across the species. Red dot indicates arginine at position 77 shown to undergo proteolytic cleavage. The amino acid sequence of the C-terminal elastin-binding domain is indicated in $\mathbf{b}$. GenBank accession numbers used to obtain primary sequences are Human (CAB38568), Orangutan (NP_001125375), Bovine (NP 001014946), Mouse (NP 035942), Rat (NP 062026), Chicken (XP 421423), Xenopus (NP 001025619) and Danio (NP 001005979)
Repeating cbEGF motifs are present in ECM and transmembrane proteins and are involved in protein-protein interactions (Maurer and Hohenester 1997). Various binding partners for fibulin-5 have been reported, including fibulin-5 itself (Jones et al. 2009; Zheng et al. 2007) (Table 1). Most notably, fibulin-5 binds to several molecules, critical for elastic fiber assembly, which will be discussed later in this review. All class II fibulins except fibulin-7 were shown to bind to tropoelastin (Kobayashi et al. 2007) and are responsible for different aspects of elastic fiber development in vivo (McLaughlin et al. 2006; McLaughlin et al. 2007). Although a direct binding of $\mathrm{Ca}^{2+}$ to the cbEGF domains of fibulin-5 has not been demonstrated, reducing the $\mathrm{Ca}^{2+}$ concentration significantly decreased tropoelastin binding to fibulin-5 in solid-phase binding assays (Wachi et al. 2007; Yanagisawa et al. 2002). Furthermore, a large deletion of the cbEGF domains of fibulin-5 exhibited a significant reduction in tropoelastin binding, suggesting that cbEGF domains play a significant role in tropoealstin-fibulin-5 binding (Zheng et al. 2007). Fibulin-5 also binds to extracellular-type superoxide dismutase (SOD3), which regulates extracellular superoxide anion $\left(\mathrm{O}_{2}^{-}\right)$levels by facilitating the conversion of $\mathrm{O}_{2}^{-}$to hydrogen peroxide $\left(\mathrm{H}_{2} \mathrm{O}_{2}\right)$ and protecting the formation of peroxynitrite. Consistent with this observation, Fbln5-null aorta was shown to contain higher amounts of $\mathrm{O}_{2}{ }^{-}$compared to wild-type controls because of the impaired tethering of SOD3 (Nguyen et al. 2004).

Fibulin-5 was shown to undergo partial proteolytic cleavage at arginine at position 77 in the N-terminal region, creating a truncated form of fibulin-5 (Hirai et al. 2007b). A point mutation of arginine to alanine inhibited proteolytic cleavage of fibulin-5 in vitro. The truncated fibulin-5 increased with aging in the skin of wild-type mice, and showed impaired ability to assemble elastic fibers in in vitro elastogenic assays. The putative protease responsible for the cleavage was suggested to be a serine protease (Hirai et al. 2007b); however, the in vivo significance of this cleavage and its relationship with human skin diseases still needs to be established.

\section{Fibulin-5 and elastic fiber development}

Elastic fibers are composed of an amorphous-appearing elastin core and a peripheral mantle of $10-\mathrm{nm}$ fibrillincontaining microfibrils. In addition to elastin, fibrillin-1, and fibrillin-2, more than 30 microfibril- and elastinassociated proteins, have been identified, including fibulins, microfibril-associated glycoproteins MAGP-1 and MAGP2 , and latent TGF- $\beta$ binding proteins- 1 through -4 (LTBPs) (reviewed in Kielty et al. 2002). Elastogenesis occurs through a series of highly regulated steps that involve secretion of the tropoelastin monomer, self-aggregation of tropoelastin, called coacervation, correct assembly and cross-linking of tropoealstin, and final organization of the insoluble elastin into functional fibers. In the mouse aorta, elastin expression starts during mid-gestation in vascular smooth muscle cells and continues for approximately 1 month after birth (Kelleher et al. 2004). Fbln 5 expression coincides with elastin expression in the aorta. During this time, the secreted elastin is cross-linked and organized into insoluble elastin sheets or laminae. Interestingly, microfibril bundles form first in the regions where elastic fibers will develop, and insoluble elastin is never observed in the 
Table 1 Interacting partners of Fibulin-5

\begin{tabular}{|c|c|c|c|}
\hline Interacting proteins & Binding site(s) within Fibulin-5 & Cellular and/or Biological functions & Reference \\
\hline \multirow{4}{*}{$\begin{array}{l}\text { Fibulin-5 } \\
\text { Tropoelastin }\end{array}$} & ND & \multirow[t]{2}{*}{ Unknown } & Zheng et al. 2007 \\
\hline & cbEGF domains & & Jones et al. 2009 \\
\hline & N-terminal cbEGF domains & \multirow[t]{2}{*}{ Elastic fiber assembly } & \multirow[t]{2}{*}{ Zheng et al. 2007} \\
\hline & C-terminal EB domain & & \\
\hline \multirow[t]{2}{*}{ Loxl-1 } & a. C-terminal fibulin domain $>1$ st cbEGF & \multirow[t]{2}{*}{ Elastic fiber assembly } & a. Hirai et al. $2007 \mathrm{a}$ \\
\hline & b. C-terminal region (245-448) & & b. Liu et al. 2004 \\
\hline Loxl-2, 4 & C-terminal fibulin domain & Elastic fiber assembly & Hirai et al. $2007 \mathrm{a}$ \\
\hline Emilin-1 & ND & Elastic fiber assembly & Zanetti et al. 2004 \\
\hline LTBP-2 & 6th cbEGF & Elastic fiber assembly & Hirai et al. $2007 b$ \\
\hline Fibrillin-1 & ND & Elastic fiber assembly & Freeman et al. 2005 \\
\hline SOD3 & C-terminal region $(320-448)$ & Superoxide scavenge & Nguyen et al. 2004 \\
\hline$\alpha 5 \beta 1, \alpha 4 \beta 1$ & N-terminal half containing RGD & $\begin{array}{l}\text { Cell attachment, Antagonize } \\
\text { fibronectin function }\end{array}$ & Lomas et al. 2007 \\
\hline$\alpha \mathrm{v} \beta 3, \alpha \mathrm{v} \beta 5$ & N-terminal half containing RGD & Cell attachment & Nakamura et al. 2002 \\
\hline$\alpha 9 \beta 1$ & ND & Cell attachment & Nakamura et al. 2002 \\
\hline Lipoprotein(a) & C-terminal domain (350-448) & Unknown & Kapetanopoulos et al. 2002 \\
\hline
\end{tabular}

$N D$ not determined

absence of microfibrils. Recently, double knockout mice for the genes encoding fibrillin- 1 (Fbn 1$)$ and fibrillin-2 (Fbn2) were generated. These mice exhibited a severe disruption of elastic fibers and a marked dysregulation of TGF- $\beta$ mediated signals (Carta et al. 2006). The latter event occurs due to the inability to tether proTGF- $\beta$ onto microfibrils via the interaction between large latency complex and fibrillin1 (Charbonneau et al. 2004; Isogai et al. 2003; Neptune et al. 2003); however, the elastin core does not seem to be involved in this process. These observations, together with biochemical and ultrastructural studies, have led to the suggestion that an interaction between microfibril proteins and tropoelastin may be critical for proper elastic fiber assembly.

\section{Role of fibulin-5 in elastic fiber assembly}

A dose-dependent, direct interaction between fibulin-5 and tropoelastin was demonstrated by solid-phase binding assays, and co-localization of fibulin-5 and elastic fibers was revealed at an electron microscopic level, providing a basis for the molecular function of fibulin-5 (Yanagisawa et al. 2002). Fibulin-5 was shown to bind tropoelastin preferentially, but not polymerized $\alpha$-elastin, suggesting its role in an early step of elastogenesis (Zheng et al. 2007). Using full-length recombinant fibulin-5, it was shown that fibulin-5 accelerates coacervation (Hirai et al. 2007b; Wachi et al. 2008), and the interaction between fibulin-5 and tropoelastin is enhanced by increased temperature and sodium chloride concentrations, which is consistent with the conditions for the efficient coacervation in vitro (Wachi et al. 2008). Furthermore, Cirulis et al. showed that fibulin5 limits the maturation of coacervated elastin fragments (Cirulis et al. 2008). Consistent with these data, Choi et al. showed by using electron microscopy that the average size of elastin aggregates was increased in the skin of Fbln5-null mice, compared with wild-type mice (Choi et al. 2009). Taken together, these findings show that fibulin- 5 functions at a formation and maturation step of coacervation to 1) control coacervation efficiency, and 2) to regulate the size of self-aggregates to achieve optimal cross-linking of tropoelastin during elastic assembly.

It was also demonstrated that fibulin-5 binds to fibrillin1, a major component of microfibrils (El-Hallous et al. 2007; Freeman et al. 2005). Fibulin-5 co-localizes with microfibrils in elastogenic cells, as well as in nonelastogenic cells, engineered to overexpress tropoelastin and fibulin-5 (Hirai et al. 2007b; Nonaka et al. 2009; Zheng et al. 2007). Consistent with these observations, a significant alteration of fibulin-5 expression was observed in tight skin mice, in which a Fbn1 mutation caused abnormally tight skin, dermal fibrosis, and impaired elastogenesis (Lemaire et al. 2004). Furthermore, it was shown that knocking down $F b n 1$ in human skin fibroblasts abrogates the formation of elastic fibers and decreases fibulin-5 immunoreactivity. Binding between LTBP-2 and fibulin-5 was shown to promote the deposition of fibulin-5 onto fibrillin-1 microribrils (Hirai et al. 2007a). Whether the formation of a fibrillin-1-fibulin-5-tropoelastin ternary complex is necessary to bind microfibrils, or whether 
fibulin-5-tropoelastin binding alone is sufficient, needs to be determined.

Fibulin-5 also binds cross-linking enzymes, including lysyl oxidase like (Loxl)-1, -2 , and -4 (Hirai et al. 2007b; Liu et al. 2004). Overexpression of Fbln 5 increased deposition of elastin and the desmosine level of elastin, indicating that fibulin-5 facilitates cross-linking of tropoelastin (Nonaka et al. 2009). Retrovirus- or adenovirus- mediated gene transfer of $F b \ln 5$ accelerated deposition of elastic fibers in human skin fibroblasts (Katsuta et al. 2008) and regenerated elastic fibers in the skin of Fbln5-null mice, respectively (Zheng et al. 2007). The deletion mutant of fibulin-5 lacking its N-terminal region, however, was unable to rescue elastic fiber defects both in vitro and in vivo, despite the presence of the elastin-binding domain located within the C-terminal fibulin module (Hirai et al. 2007b; Zheng et al. 2007). Therefore, the C-terminal elastin-binding domain is required but not sufficient for the formation of elastic fibers.

Although the significance of the RGD sequence in elastic fiber assembly was initially suggested, generation of mutant mice expressing fibulin-5 D56E ( Fbln $\left.5^{R G E / R G E}\right)$, a mutation known to disrupt the binding of the ECM to RGD-dependent integrins (Yang et al. 2007), developed completely normal elastic fibers (our unpublished observation), suggesting that cell-surface binding of fibulin-5 mediated by integrins is dispensable for the formation of elastic fibers. Taken together, we propose a model in which fibulin-5 binds tropoelastin to regulate the coacervation step and serves as an adaptor to bind the cross-linking enzymes, tropoelastin and microfibrils, to aid in elastic fiber assembly (Fig. 3).

Mutation of fibulin-5 in human elastic fiber disease

Human genetic studies have identified two homozygous missense mutations of FBLN5 (p.S227P) and (p.C217R) in autosomal recessive (AR) cutis laxa families (Claus et al. 2008; Elahi et al. 2006; Loeys et al. 2002). The first mutation was reported in two families of ethnically different groups and the disease manifested as a severe form of cutis laxa with internal organ involvement (AR cutis laxa type I). Biochemically, both substitutions of fibulin-5 (S227P and C217R) were shown to decrease binding to tropoelastin. The S227P mutation further revealed a significant decrease in synthesis and secretion of the mutant protein and impaired association with fibrillin-1 $(\mathrm{Hu}$ et al. 2006). A heterozygous tandem duplication of fibulin-5 was also reported in a patient with a mild form of cutis laxa, suggesting that the large mutant protein might act in a dominant negative fashion (Markova et al. 2003).
Heterozygous missense variations in FBLN5 were reported to be associated with age-related macular degeneration (ARMD), a common cause of progressive vision loss (Lotery et al. 2006; Stone et al. 2004). Fibulin-5 is normally localized in Bruch's membrane and the choriocapilaris of the retina, where elastic fibers are present. Fibulin-5 was observed in the pathological basal deposits beneath the retinal pigment epithelium in eyes affected by age-related macular degeneration (Mullins et al. 2007). Some mutations of fibulin-5 (G412E, G267S, I169 T, and Q124P) were shown to cause decreased secretion of the mutant protein into the media (Lotery et al. 2006), suggesting compromised elastic fiber formation as the underlying mechanism. However, the causal relationship between heterozygous missense mutations in fibulin-5 and ARMD needs to be further investigated.

Alteration of fibulin-5 in pathological conditions involving elastic fibers

Fbln5-null mice were shown to develop pelvic organ prolapse and have served as one of the first animal models for this condition (Drewes et al. 2007). By 6 months of age, $92 \%$ of $F b \ln 5$-null females developed vaginal prolapse with severe disruptions in elastic fiber formation. Biomechanical studies using vagina tissue from pregnant and non-pregnant females from wild-type and Fbln5-null mice revealed that the Fbln5-null vagina with prolapse was similar to that of pregnant wild-type females, exhibiting a significant decrease in vaginal stiffness (Rahn et al. 2008). Subsequently, it was shown in the uterosacral ligaments of patients with pelvic organ prolapse (POP) that expression of fibulin-5 was significantly decreased (Jung et al. 2009). Additionally, fibulin-5 mRNA was decreased in para-urethral biopsies obtained from POP women (Soderberg et al. 2009), underscoring defective elastic fibers as an underlying cause of POP.

Another organ affected by the absence of fibulin- 5 is the lung, in which the defect is manifested as an emphysematous lung that progressively worsens after birth. Expression of fibulin-5 is observed during embryogenesis and continues after birth to complete the development of the lung. Interestingly, FGF18 expression, which peaks after birth, correlates with the expression of tropoelastin in vivo, and supports elastogenesis by inducing proliferation of lung fibroblasts, as well as by expression of elastogenic genes, including Fbln 5 and Lox (Chailley-Heu et al. 2005). Fbln 5 expression was confirmed in rat lung interstitial fibroblasts, and was markedly increased after treatment with TGF- $\beta$ or following elastase-induced lung injury (Kuang et al. 2003; Kuang et al. 2006). On the other hand, treatment of lung interstitial fibroblasts with interleukin $-1 \beta$ completely abolished the expression of $F b \ln 5$ (Kuang et al. 2003), demonstrating the regulation of $F b \ln 5$ by distinct sets of cytokines. 


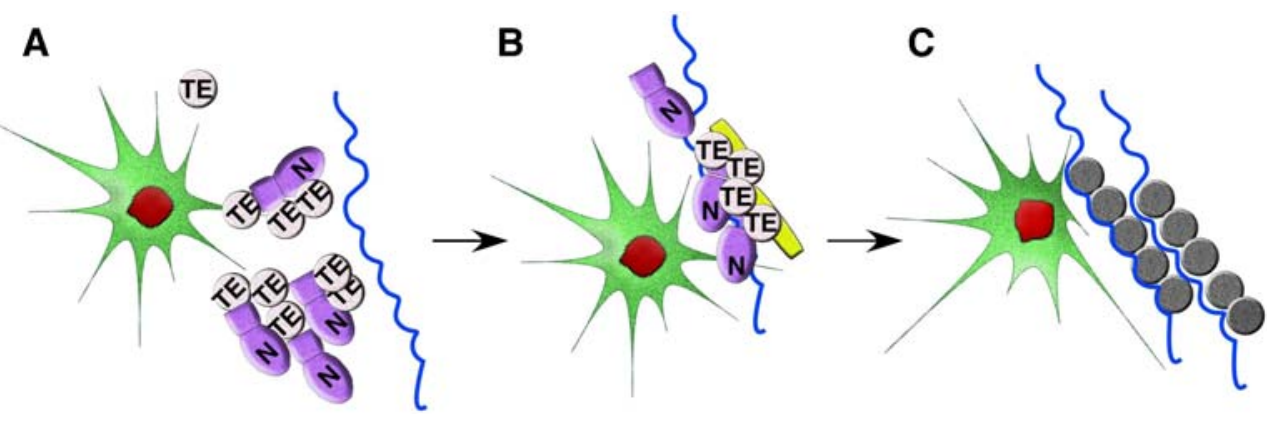

Fig. 3 A model of elastic fiber assembly. a Secretion of tropoelastin (TE) from elastogenic cells (green). Tropoelastin undergoes selfaggregation and fibulin-5 (purple) mediates this process. Tropoealstin binds both $\mathrm{N}$-terminal $(N)$ and $\mathrm{C}$-terminal tropoelastin-binding

\section{Effect of fibulin-5 on vascular cells}

The function of fibulin-5 in adult blood vessels was suggested by the strong upregulation of Fbln 5 in the neointima after balloon withdrawal injury or carotid artery ligation, and in activated endothelial cells of atherosclerotic plaques (Kowal et al. 1999; Spencer et al. 2005). Fbln5-null SMCs displayed enhanced proliferation and migration in response to serum and PDGF. This enhancement was inhibited by over-expression of Fbln 5 (Spencer et al. 2005). Forced expression of Fbln5 in primary human endothelial cells improved cell attachment against continuous shear stress and enhanced cell retention on artificial grafts subjected to pulsatile flow. However, the proliferation of endothelial cells was decreased in Fbln5-overexpressing cells compared to control cells (Preis et al. 2006). Human primary SMCs plated on recombinant fibulin-5 exhibited PDGF receptors with undetectable phosphorylation, and poorly phosphorylated EGF receptors, compared to SMCs plated on fibronectin (Lomas et al. 2007). Interestingly, the presence of integrin $\beta 1$-activating antibody in SMCs, plated on fibulin-5, restored phosphorylation of PDGF $\alpha$ and $\beta$ receptors. Together with the observation that fibulin-5 binds fibronectin receptors $(\alpha 5 \beta 1$ and $\alpha 4 \beta 1)$ but fails to activate downstream signaling, these data suggest a possibility that fibulin-5 may regulate vascular cell behavior by antagonizing fibronectin-mediated signaling. However, further investigation is required to establish the in vivo relevance of the fibulin-5-integrin binding during vessel development and pathological insults.

\section{Fibulin-5 and angiogenesis}

The effect of fibulin-5 on angiogenesis was first described using mouse brain microvascular MB114 endothelial cells, in which Fbln 5 overexpression or incubation with recombinant fibulin-5 inhibited sprouting, proliferation, and domains (square) of fibulin-5. b Fibulin-5 binds tropoelastin, microfibrils (blue), and lysyl oxidase-like enzyme (yellow) to aid in elastic fiber assembly. c Cross-linked insoluble elastin (grey) is polymerized and organized into functional elastic fibers

invasion in matrigel (Albig and Schiemann 2004). Upregulation of thrombospondin-1 expression, as well as antagonization of $\mathrm{VEGF}_{165}$-mediated signaling, including activation of P38 MAPK and ERK1/2, were suggested to be the underlying causes of the inhibitory effect of fibulin5. Fibulin-5 was also shown to reduce migration of human microvascular HMEC-1 endothelial cells toward fibronectin, and overexpression of FBLN5 in MB114 cells downregulated MMP-2 expression, as well as enzymatic activity during tubulogenesis on collagen gels (Albig et al. 2006). Matrigel implantation experiments in the skin of wild-type mice showed that matrigel containing high or low doses of fibulin-5 stimulated the invasion of bFGF-induced fibroblasts, but inhibited vessel formation and angiogenesis, independent of RGD-integrin interactions. Consistent with these observations, Fbln 5 -null mice exhibited increased angiogenesis after physiological wound healing and PVA sponge implantation, without an effect on fibroblast invasion (Sullivan et al. 2007; Zheng et al. 2006).

Although exogenous and endogenous fibulin-5 was shown to antagonize angiogenesis, the exact mechanism behind this effect has remained elusive. We recently observed that fibulin-5 exerts its effect on endothelial cells and angiogenesis by controlling integrin-induced production of reactive oxygen species (ROS), which have proangiogenic properties (Schluterman et al. 2009). ROS, including $\mathrm{O}_{2}^{-}$and $\mathrm{H}_{2} \mathrm{O}_{2}$, are highly reactive molecules produced commonly as by products of aerobic respiration and the mitochondrial transport chain. ROS were originally identified as host defense molecules produced by neutrophils via $\mathrm{NAD}(\mathrm{P}) \mathrm{H}$ oxidases, and have a critical function in eliciting biological processes required for the initiation of angiogenesis ( $\mathrm{Wu}$ 2006). By functioning as signaling molecules, ROS have been shown to activate pathways such as proliferation, cell adhesion, motility and invasion in endothelial cells (Ushio-Fukai 2007).

Endothelial cells, treated with $\mathrm{H}_{2} \mathrm{O}_{2}$, produced higher levels of VEGF, thereby increasing their proliferation and 
migration (Chua et al. 1998). It has also been shown that ROS, produced in response to hypoxic conditions, can facilitate capillary tube formation in human microvascular endothelial cells, and this process was inhibited by treatment with an anti-oxidant (Lelkes et al. 1998). ROS have also been shown to stimulate angiogenesis in vivo. For example, neovascularization in response to hindlimb ischemia was significantly impaired in mice lacking gp91phox, a critical component of NADPH oxidases (Tojo et al. 2005). However, the production of ROS must be tightly regulated because a large excess of ROS can be detrimental to the remodeling process and result in endothelial cell death (Touyz and Schiffrin 2004). Since $\alpha 5 \beta 1$ integrin serves as the primary fibronectin receptor and stimulates ROS production (Chiarugi et al. 2003), and fibulin-5 binding to $\alpha 5 \beta 1$ leads to inhibition of fibronectin-mediated downstream signaling (Lomas et al. 2007), it is plausible that fibulin-5 antagonizes endothelial cell proliferation and migration by controlling ROS levels through binding to $\alpha 5 \beta 1$ integrin. Interestingly, ROS production increases in response to vascular injury and atherosclerotic changes as part of the inflammatory response to aid in endothelial cell stimulation (Cai and Harrison 2000). Therefore, it is possible that the increase in fibulin-5 expression observed following trauma to the vasculature could be used as a mechanism to control ROS production during such events.

\section{Fibulin-5 in tumorigenesis}

Given the varying effects of fibulin- 5 on different cell populations, it is no surprise that the effect of fibulin-5 on tumor growth is complex and appears to be largely contextdependent. For example, MCA 102 fibrosarcoma cells, stably expressing $F b \ln 5$ and subcutaneously injected into isogenic wild-type mice, produced significantly reduced tumor growth, despite the fact that stimulation with fibulin-5 increased the invasiveness of MCA 102 cells into a synthetic basement membrane in vitro (Albig et al. 2006). When Fbln5overexpressing HT1080 fibrosarcoma cells were injected into BALB/c SCID mice, tumor growth was inhibited and tumor blood vessel formation was significantly decreased compared to the mice injected with control HT1080 cells (Xie et al. 2008). In stark contrast, overexpression of Fbln 5 in $4 \mathrm{~T} 1$ breast cancer cells increased invasiveness by inducing TGF- $\beta$-stimulated epithelial-mesenchymal transitions and MMP expression. These alterations led to increased tumor growth in vivo when these cells were implanted into normal wild-type mice. (Lee et al. 2008).

Examination of FBLN5 mRNA levels in various human tumors from kidney, breast, prostate, lung and gastrointestinal organs, revealed that FBLN5 expression was down-regulated in $62 \%$ of tumors in comparison to control normal tissues
(Schiemann et al. 2002). However, the analysis of FBLN5 expression in human tumors has been limited and has largely centered on whole tumor tissue analysis, allowing for no distinction between tumor cell-derived and host-derived fibulin-5. Given the differential effects of fibulin-5 on cells of epithelial origin and on cells of mesenchymal origin (Schiemann et al. 2002), the overall effect of fibulin-5 on tumor development must be carefully evaluated in human tumor specimens.

\section{Conclusions and future directions}

Fibulin-5 is a matricellular protein, contributing to the structural development of elastogenic tissues, as well as mediating various cellular functions required for the maintenance of tissue homeostasis. The discovery of fibulin-5 has provided a new insight into the regulated steps of elastic fiber assembly and has given us an opportunity to explore therapeutic implications, including prevention of elastic fiber-degenerative conditions, regeneration of damaged elastic fibers, and development of efficient artificial blood vessels. The availability of genetically engineered mice that enables us to delete $F b \ln 5$ after the completion of elastic fibers will allow us to distinguish between the elastic fiber-dependent or -independent effect of fibulin-5. It also remains to be investigated how Class II fibulins are involved in elastic fiber development in vivo, and what are the redundant and specific roles among these fibulins. A diverse cellular effect of fibulin-5, in a cell-type specific and context-dependent manner, suggests that there may be multiple fibulin-5-interacting proteins at the cell surface, including integrins. It remains to be determined how fibulin-5 mediates or antagonizes cellular signaling at the cell surface, and whether modulation of fibulin- 5 has the potential for the therapy of angiogenesis-dependent pathologies.

Acknowledgment We thank all the members of Yanagisawa and Brekken laboratories for discussion. Technical expertise of the Pathology Core Laboratory and Transgenic Core laboratory at the University of Texas Southwestern Medical Center is greatly acknowledged. Research support was provided in part by the National Institutes of Health (HL071157, AG028048, CA118240), The Welch Foundation and American Heart Association South Central Affiliate.

Open Access This article is distributed under the terms of the Creative Commons Attribution Noncommercial License which permits any noncommercial use, distribution, and reproduction in any medium, provided the original author(s) and source are credited.

\section{References}

Albig AR, Schiemann WP (2004) Fibulin-5 antagonizes vascular endothelial growth factor (VEGF) signaling and angiogenic sprouting by endothelial cells. DNA \& Cell Biology 23:367-379 
Albig AR, Schiemann WP (2005) Fibulin-5 function during tumorigenesis. Future Oncol 1:23-35

Albig AR, Neil JR, Schiemann WP (2006) Fibulins 3 and 5 antagonize tumor angiogenesis in vivo. Cancer Res 66:26212629

Argraves WS, Greene LM, Cooley MA, Gallagher WM (2003) Fibulins: physiological and disease perspectives. EMBO Rep 4:1127-1131

Bornstein P (1995) Diversity of function is inherent in matricellular proteins: an appraisal of thrombospondin 1. J Cell Biol 130:503506

Bornstein P (2001) Thrombospondins as matricellular modulators of cell function. J Clin Invest 107:929-934

Brekken RA, Sage EH (2001) SPARC, a matricellular protein: at the crossroads of cell-matrix communication. Matrix Biol 19:816827

Cai H, Harrison DG (2000) Endothelial dysfunction in cardiovascular diseases: the role of oxidant stress. Circ Res 87:840-844

Carta L, Pereira L, Arteaga-Solis E, Lee-Arteaga SY, Lenart B, Starcher B et al (2006) Fibrillins 1 and 2 perform partially overlapping functions during aortic development. J Biol Chem 281:8016-8023

Chailley-Heu B, Boucherat O, Barlier-Mur AM, Bourbon JR (2005) FGF-18 is upregulated in the postnatal rat lung and enhances elastogenesis in myofibroblasts. Am J Physiol Lung Cell Mol Physiol 288:L43-51

Charbonneau NL, Ono RN, Corson GM, Keene DR, Sakai LY (2004) Fine tuning of growth factor signals depends on fibrillin microfibril networks. Birth Defects Res C Embryo Today $72: 37-50$

Chiarugi P, Pani G, Giannoni E, Taddei L, Colavitti R, Raugei G et al (2003) Reactive oxygen species as essential mediators of cell adhesion: the oxidative inhibition of a FAK tyrosine phosphatase is required for cell adhesion. J Cell Biol 161:933-944

Choi J, Bergdahl A, Zheng Q, Starcher B, Yanagisawa H, Davis EC (2009) Analysis of dermal elastic fibers in the absence of fibulin5 reveals potential roles for fibulin-5 in elastic fiber assembly. Matrix Biol 28:211-220

Chu ML, Tsuda T (2004) Fibulins in development and heritable disease. Birth Defects Res C Embryo Today 72:25-36

Chua CC, Hamdy RC, Chua BH (1998) Upregulation of vascular endothelial growth factor by $\mathrm{H} 2 \mathrm{O} 2$ in rat heart endothelial cells. Free Radic Biol Med 25:891-897

Cirulis JT, Bellingham CM, Davis EC, Hubmacher D, Reinhardt DP, Mecham RP et al (2008) Fibrillins, fibulins, and matrixassociated glycoprotein modulate the kinetics and morphology of in vitro self-assembly of a recombinant elastin-like polypeptide. Biochemistry 47:12601-12613

Claus S, Fischer J, Megarbane H, Megarbane A, Jobard F, Debret R et al (2008) A p.C217R mutation in fibulin-5 from cutis laxa patients is associated with incomplete extracellular matrix formation in a skin equivalent model. J Invest Dermatol 128:1442-1450

Davis GE, Bayless KJ, Davis MJ, Meininger GA (2000) Regulation of tissue injury responses by the exposure of matricryptic sites within extracellular matrix molecules. Am J Pathol 156:14891498

de Vega S, Iwamoto T, Nakamura T, Hozumi K, McKnight DA, Fisher LW et al (2007) TM14 is a new member of the fibulin family (fibulin-7) that interacts with extracellular matrix molecules and is active for cell binding. J Biol Chem 282:30878-30888

de Vega S, Iwamoto T, Yamada Y (2009) Fibulins: Multiple roles in matrix structures and tissue functions. Cell Mol Life Sci In Press

Drewes PG, Yanagisawa H, Starcher B, Hornstra IK, Csiszar K, Marinis SI et al (2007) Pelvic organ prolapse in Fibulin-5 knockout mice: pregnancy changes in elastic fiber homeostasis in mouse vagina. Am J Pathol 170:578-589

El-Hallous E, Sasaki T, Hubmacher D, Getie M, Tiedemann K, Brinckmann $\mathrm{J}$ et al (2007) Fibrillin-1 interactions with fibulins depend on the first hybrid domain and provide an adaptor function to tropoelastin. J Biol Chem 282:8935-8946

Elahi E, Kalhor R, Banihosseini SS, Torabi N, Pour-Jafari H, Houshmand $\mathrm{M}$ et al (2006) Homozygous missense mutation in fibulin-5 in an Iranian autosomal recessive cutis laxa pedigree and associated haplotype. J Invest Dermatol 126:1506-1509

Freeman LJ, Lomas A, Hodson N, Sherratt MJ, Mellody KT, Weiss AS et al (2005) Fibulin-5 interacts with fibrillin-1 molecules and microfibrils. Biochem J 388:1-5

Hirai M, Horiguchi M, Ohbayashi T, Kita T, Chien KR, Nakamura T (2007a) Latent TGF-beta-binding protein 2 binds to DANCE/ fibulin-5 and regulates elastic fiber assembly. EMBO J 26:32833295

Hirai M, Ohbayashi T, Horiguchi M, Okawa K, Hagiwara A, Chien KR et al (2007b) Fibulin-5/DANCE has an elastogenic organizer activity that is abrogated by proteolytic cleavage in vivo. J Cell Biol 176:1061-1071

Hu Q, Loeys BL, Coucke PJ, De Paepe A, Mecham RP, Choi J et al (2006) Fibulin-5 mutations: mechanisms of impaired elastic fiber formation in recessive cutis laxa. Hum Mol Genet 15:3379-3386

Isogai Z, Ono RN, Ushiro S, Keene DR, Chen Y, Mazzieri R et al (2003) Latent transforming growth factor beta-binding protein 1 interacts with fibrillin and is a microfibril-associated protein. $\mathrm{J}$ Biol Chem 278:2750-2757

Jones RP, Wang M-C, Jowitt TA, Ridley C, Mellody KT, Howard M et al (2009) Fibulin 5 forms a compact dimer in physiological solutions. J Biol Chem In Press

Jung HJ, Jeon MJ, Yim GW, Kim SK, Choi JR, Bai SW (2009) Changes in expression of fibulin-5 and lysyl oxidase-like 1 associated with pelvic organ prolapse. Eur J Obstet Gynecol Reprod Biol

Karnik SK, Brooke BS, Bayes-Genis A, Sorensen L, Wythe JD, Schwartz RS et al (2003) A critical role for elastin signaling in vascular morphogenesis and disease. Development 130:411-423

Katsuta Y, Ogura Y, Iriyama S, Goetinck PF, Klement JF, Uitto J et al (2008) Fibulin-5 accelerates elastic fibre assembly in human skin fibroblasts. Exp Dermatol 17:837-842

Kapetanopoulos A, Fresser F, Millonig G, Shaul Y, Baier G, Utermann G (2002) Direct interaction of extracellular matrix protein DANCE with apolipoprotein (a) mediated by the kringle IVtype 2 domain. Mol. Genet. Genomics 267:440-460

Kelleher CM, McLean SE, Mecham RP (2004) Vascular extracellular matrix and aortic development. Curr Top Dev Biol 62:153-188

Kielty CM, Sherratt MJ, Shuttleworth CA (2002) Elastic fibres. J Cell Sci 115:2817-2828

Kobayashi N, Kostka G, Garbe JH, Keene DR, Bächinger HP, Hanisch FG et al (2007) A comparative analysis of the fibulin protein family. Biochemical characterization, binding interactions, and tissue localization. J Biol Chem 282:11805-11816

Kowal RC, Richardson JA, Miano JM, Olson EN (1999) EVEC, a novel epidermal growth factor-like repeat-containing protein upregulated in embryonic and diseased adult vasculature. Circ Res 84:1166-1176

Kuang PP, Goldstein RH, Liu Y, Rishikof DC, Jean JC, Joyce-Brady M (2003) Coordinate expression of fibulin-5/DANCE and elastin during lung injury repair. Am J Physiol Lung Cell Mol Physiol 285:1147

Kuang PP, Joyce-Brady M, Zhang XH, Jean JC, Goldstein RH (2006) Fibulin-5 gene expression in human lung fibroblasts is regulated by TGF-beta and phosphatidylinositol 3-kinase activity. Am J Physiol Cell Physiol 291:C1412-1421 
Lee YH, Albig AR, Regner M, Schiemann BJ, Schiemann WP (2008) Fibulin-5 initiates epithelial-mesenchymal transition (EMT) and enhances EMT induced by TGF-beta in mammary epithelial cells via a MMP-dependent mechanism. Carcinogenesis 29:22432251

Legate KR, Montanez E, Kudlacek O, Fassler R (2006) ILK, PINCH and parvin: the tIPP of integrin signalling. Nat Rev Mol Cell Biol $7: 20-31$

Lelkes PI, Hahn KL, Sukovich DA, Karmiol S, Schmidt DH (1998) On the possible role of reactive oxygen species in angiogenesis. Adv Exp Med Biol 454:295-310

Lemaire R, Korn JH, Schiemann WP, Lafyatis R (2004) Fibulin-2 and fibulin-5 alterations in tsk mice associated with disorganized hypodermal elastic fibers and skin tethering. J Invest Dermatol 123:1063-1069

Liu X, Zhao Y, Gao J, Pawlyk B, Starcher B, Spencer JA et al (2004) Elastic fiber homeostasis requires lysyl oxidase-like 1 protein. Nat Genet 36:178-182

Loeys B, Van Maldergem L, Mortier G, Coucke P, Gerniers S, Naeyaert JM et al (2002) Homozygosity for a missense mutation in fibulin-5 (FBLN5) results in a severe form of cutis laxa. Hum Mol Genet 11:2113-2118

Lomas AC, Mellody KT, Freeman LJ, Bax DV, Shuttleworth CA, Kielty CM (2007) Fibulin-5 binds human smooth-muscle cells through alpha5beta1 and alpha4beta1 integrins, but does not support receptor activation. Biochem J 405:417-428

Lotery AJ, Baas D, Ridley C, Jones RP, Klaver CC, Stone E et al (2006) Reduced secretion of fibulin 5 in age-related macular degeneration and cutis laxa. Hum Mutat 27:568-574

Madsen CD, Sidenius N (2008) The interaction between urokinase receptor and vitronectin in cell adhesion and signalling. Eur $\mathrm{J}$ Cell Biol 87:617-629

Markova D, Zou Y, Ringpfeil F, Sasaki T, Kostka G, Timpl R et al (2003) Genetic heterogeneity of cutis laxa: a heterozygous tandem duplication within the fibulin-5 (FBLN5) gene. Am J Hum Genet 72:998-1004

Maurer P, Hohenester E (1997) Structural and functional aspects of calcium binding in extracellular matrix proteins. Matrix Biol 15:569-580 discussion $581 \mathrm{cscs}$

McLaughlin PJ, Chen Q, Horiguchi M, Starcher BC, Stanton JB, Broekelmann TJ et al (2006) Targeted disruption of fibulin-4 abolishes elastogenesis and causes perinatal lethality in mice. Mol Cell Biol 26:1700-1709

McLaughlin PJ, Bakall B, Choi J, Liu Z, Sasaki T, Davis EC et al (2007) Lack of fibulin-3 causes early aging and herniation, but not macular degeneration in mice. Hum Mol Genet 16:3059-3070

Mullins RF, Olvera MA, Clark AF, Stone EM (2007) Fibulin-5 distribution in human eyes: relevance to age-related macular degeneration. Exp Eye Res 84:378-380

Nakamura T, Ruiz-Lozano P, Lindner V, Yabe D, Taniwaki M, Furukawa Y et al (1999) DANCE, a novel secreted RGD protein expressed in developing, atherosclerotic, and balloon-injured arteries. J Biol Chem 274:22476-22483

Nakamura T, Lozano PR, Ikeda Y, Iwanaga Y, Hinek A, Minamisawa $\mathrm{S}$ et al (2002) Fibulin-5/DANCE is essential for elastogenesis in vivo. Nature 415:171-175

Neptune ER, Frischmeyer PA, Arking DE, Myers L, Bunton TE, Gayraud B et al (2003) Dysregulation of TGF-beta activation contributes to pathogenesis in Marfan syndrome. Nat Genet $33: 407-411$

Nguyen AD, Itoh S, Jeney V, Yanagisawa H, Fujimoto M, UshioFukai $M$ et al (2004) Fibulin-5 is a novel binding protein for extracellular superoxide dismutase. Circ Res 95:1067-1074

Nonaka R, Onoue S, Wachi H, Sato F, Urban Z, Starcher BC et al (2009) DANCE/fibulin-5 promotes elastic fiber formation in a tropoelastin isoform-dependent manner. Clin Biochem 42:713-721
Preis M, Cohen T, Sarnatzki Y, Ben Yosef Y, Schneiderman J, Gluzman Z et al (2006) Effects of fibulin-5 on attachment, adhesion, and proliferation of primary human endothelial cells. Biochem Biophys Res Commun 348:1024-1033

Rahn DD, Ruff MD, Brown S, Tibbals HF, Word RA (2008) Biomechanical properties of the mouse vagina: changes seen in pregnancy and with elastinopathy. Am J Obstet Gynecol 198:590e591-596

Raines EW (2000) The extracellular matrix can regulate vascular cell migration, proliferation, and survival: relationships to vascular disease. Int J Exp Pathol 81:173-182

Ruoslahti E, Pierschbacher MD (1987) New perspectives in cell adhesion: RGD and integrins. Science 238:491-497

Schiemann WP, Blobe GC, Kalume DE, Pandey A, Lodish HF (2002) Context-specific Effects of Fibulin-5 (DANCE/EVEC) on cell proliferation, motility, and invasion. Fibulin-5 is induced by transforming growth factor-beta and affects protein kinase cascades. J Biol Chem 277:27367-27377

Schluterman MK, Chapman SL, Korpanty G, Ozumi K, Fukai T, Yanagisawa H, Brekken RA (2009) Loss of fibulin-5 binding to $\beta 1$ integrins inhibits tumor growth by increasing the level of ROS. Disease Models and Mechanisms In Press

Shen Q, Wang Y, Kokovay E, Lin G, Chuang SM, Goderie SK et al (2008) Adult SVZ stem cells lie in a vascular niche: a quantitative analysis of niche cell-cell interactions. Cell Stem Cell 3:289-300

Soderberg MW, Bystrom B, Kalamajski S, Malmstrom A, EkmanOrdeberg G (2009) Gene expressions of small leucine-rich repeat proteoglycans and fibulin-5 are decreased in pelvic organ prolapse. Mol Hum Reprod 15:251-257

Spencer JA, Hacker SL, Davis EC, Mecham RP, Knutsen RH, Li DY et al (2005) Altered vascular remodeling in fibulin-5-deficient mice reveals a role of fibulin- 5 in smooth muscle cell proliferation and migration. Proc Natl Acad Sci U S A 102:2946-2951

Stone EM, Braun TA, Russell SR, Kuehn MH, Lotery AJ, Moore PA et al (2004) Missense variations in the fibulin 5 gene and agerelated macular degeneration. N Engl J Med 351:346-353

Sullivan KM, Bissonnette R, Yanagisawa H, Hussain SN, Davis EC (2007) Fibulin-5 functions as an endogenous angiogenesis inhibitor. Lab Invest 87:818-827

ten Dijke P, Arthur HM (2007) Extracellular control of TGFbeta signalling in vascular development and disease. Nat Rev Mol Cell Biol 8:857-869

Timpl R, Sasaki T, Kostka G, Chu ML (2003) Fibulins: a versatile family of extracellular matrix proteins. Nat Rev Mol Cell Biol 4:479-489

Tojo T, Ushio-Fukai M, Yamaoka-Tojo M, Ikeda S, Patrushev N, Alexander RW (2005) Role of gp91phox (Nox2)-containing $\mathrm{NAD}(\mathrm{P}) \mathrm{H}$ oxidase in angiogenesis in response to hindlimb ischemia. Circulation 111:2347-2355

Touyz RM, Schiffrin EL (2004) Reactive oxygen species in vascular biology: implications in hypertension. Histochem Cell Biol 122:339-352

Ushio-Fukai M (2007) VEGF signaling through NADPH oxidasederived ROS. Antioxid Redox Signal 9:731-739

Vogel BE, Hedgecock EM (2001) Hemicentin, a conserved extracellular member of the immunoglobulin superfamily, organizes epithelial and other cell attachments into oriented line-shaped junctions. Development 128:883-894

Wachi H, Sato F, Nakazawa J, Nonaka R, Szabo Z, Urban Z et al (2007) Domains 16 and 17 of tropoelastin in elastic fibre formation. Biochem J 402:63-70

Wachi H, Nonaka R, Sato F, Shibata-Sato K, Ishida M, Iketani S et al (2008) Characterization of the molecular interaction between tropoelastin and DANCE/fibulin-5. J Biochem 143:633-639 
Wu C, Keivens VM, O'Toole TE, McDonald JA, Ginsberg MH (1995) Integrin activation and cytoskeletal interaction are essential for the assembly of a fibronectin matrix. Cell 83:715-724

$\mathrm{Wu}$ WS (2006) The signaling mechanism of ROS in tumor progression. Cancer Metastasis Rev 25:695-705

Xie L, Palmsten K, MacDonald B, Kieran MW, Potenta S, Vong S et al (2008) Basement membrane derived fibulin-1 and fibulin-5 function as angiogenesis inhibitors and suppress tumor growth. Exp Biol Med (Maywood) 233:155-162

Yanagisawa H, Davis EC, Starcher BC, Ouchi T, Yanagisawa M, Richardson JA et al (2002) Fibulin-5 is an elastin-binding protein essential for elastic fibre development in vivo. Nature 415:168-171

Yang Z, Mu Z, Dabovic B, Jurukovski V, Yu D, Sung J et al (2007) Absence of integrin-mediated TGFbetal activation in vivo recapitulates the phenotype of TGFbeta1-null mice. J Cell Biol 176:787-793

Zanetti M, Braghetta P, Sabatelli P, Mura I, Doliana R, Colombatti A, Volpin D, Bonaldo P, Bressan GM (2004) Emilin-1 deficiency induces elastogenesis and vascular cell defects. Mol. Cell. Biol 24:638-650

Zheng Q, Choi J, Rouleau L, Leask RL, Richardson JA, Davis EC et al (2006) Normal Wound Healing in Mice Deficient for Fibulin-5, an Elastin Binding Protein Essential for Dermal Elastic Fiber Assembly. $\mathrm{J}$ Invest Dermatol

Zheng Q, Davis EC, Richardson JA, Starcher BC, Li T, Gerard $\mathrm{RD}$ et al (2007) Molecular analysis of fibulin-5 function during de novo synthesis of elastic fibers. Mol Cell Biol 27:1083-1095 